\title{
Aplicación de proceso de Enfermería: estudio de caso de una paciente gestante de 31.6 semanas, rotura prematura de membranas y embarazo gemelar
}

\author{
Applying nursing process: a case study of a pregnant patient of 31.6 weeks, premature rupture of \\ membranes and twin pregnancy
}

Ruth Chura Muñuico

\begin{abstract}
RESUMEN
La Rotura Prematura de Membranas (RPM) es una complicación del embarazo que causa el $40 \%$ de los partos prétermino y conlleva el $10 \%$ de la mortalidad neonatal. El presente estudio tuvo como objetivo implementar el proceso de Enfermería en el cuidado de una gestante de 31.6 semanas por FUR, Rotura Prematura de Membranas, embarazo gemelar, antecedentes de fertilización in Vitrio e ITU en tratamiento, del Servicio de Ginecobstetricia Sala "B" del Hospital Nacional Docente Madre Niño San Bartolomé en el período de noviembre - diciembre 2011. Los datos fueron obtenidos después de la valoración por patrones funcionales de Marjory Gordon, posteriormente se encontraron diagnósticos de la Taxonomía II NANDA International 2012 - 2014 fundamentados en el modelo de Virginia Herdenson. Se estandarizan los siguientes diagnósticos enfermeros para el plan de cuidados: Riesgo de alteración de la diada materno fetal. Perfusión tisular periférica ineficaz. Riesgo de infección. Dolor agudo. Ansiedad. Deterioro de la movilidad física. Trastorno del patrón de sueño. Déficit de autocuidado: baño, uso de inodoro. Riesgo de deterioro de la integridad cutánea. Riesgo de motilidad gastrointestinal disfuncional. Disposición para mejorar la gestión de la propia salud. El proceso de atención de Enfermería es importante porque tiene repercusión sobre la profesión, la paciente y la enfermera; profesionalmente el proceso define el foco del trabajo enfermero en la práctica clínica, con la perspectiva de favorecer la calidad de vida de la paciente a través de los cuidados de calidad, y así seguir consolidando el trabajo de la Enfermería.
\end{abstract}

Palabras clave: Rotura Prematura Membranas, embarazo gemelar, proceso de atención de enfermería.

\begin{abstract}
Premature Rupture of Membranes (PROM) is a complication of pregnancy that causes $40 \%$ of pre-term births and carries $10 \%$ of neonatal mortality. This study aimed to implement the nursing process in the care of a 31.6 week old pregnant woman, Premature Rupture of Membranes, Twin pregnancy, history of fertilization in vitrio and UTI treatment, in Obstetrics and Gynecology Service Room "B" National Teaching Hospital Child Mother St. Bartholomew in the period November -December 2011. Data was obtained after the evaluation of Marjory Gordon's functional patterns, later diagnosed by The NANDA Taxonomy II International 2012 - 2014, a model founded by Virginia Herdenson. The following nursing diagnoses for standardized care plan are as follows: Risk of impaired fetal maternal Dyad, Ineffective peripheral tissue perfusion, Risk of infection, Acute pain, Anxiety, Impaired physical mobility, sleep pattern disorder, Self-care deficit: bathing, toilet use, Risk for impaired skin integrity, Risk for dysfunctional gastrointestinal motility and Willingness to better manage their own health. The process of nursing care is important because it has an impact on the profession, the patient and nurse; Professionally process defines the focus of nurses working in clinical practice, with a view to enhance the quality of life of the patient through quality care and thus further strengthen the work of nursing.
\end{abstract}

Keywords: Premature Rupture Membranes, twin pregnancy, nursing care process

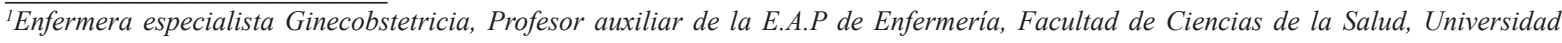
Peruana Unión, Lima.

Correspondencia: ruhis@upeu.edu.pe 


\section{INTRODUCCIÓN}

\section{Rotura Prematura de Membranas}

La Rotura Prematura de Membranas (RPM) ovulares, se entiende como la pérdida de la integridad del saco ovular, con la consiguiente salida de líquido amniótico (LA) y la puesta en comunicación de la cavidad amniótica con el canal endocervical y la vagina. Sucede antes del inicio del parto, y con independencia de la edad gestacional. Usandizaga (2005).

Según Cararach 2006, la época de gestación se divide en:

- RPM pretérmino. Antes de la semana 37 de gestación.

- RPM a término. Posterior a la semana 36 completa de gestación.

- A la hora de orientar el tratamiento, se clasifican según la semana de gestación en la que se produjo la rotura.

- Mayor o igual a 35 semanas: Fetos maduros.

- Entre 26 y 34 semanas: Fetos inmaduros.

- Menor a 26 semanas: Fetos previables.

\section{Etiología}

Vivanco (2012) refiere que las membranas fetales están constituidas por una capa interna denominada amnios y otra capa externa denominada corion. Están separadas por tejido conectivo denso que contiene una pequeña proporción de elastina. Tanto el colágeno como la elastina tienen un papel fundamental en la integridad de la membrana corioamniótica. Ambas están protegidas por el músculo uterino y el soporte mecánico que supone el cuello del útero cerrado. En condiciones normales, la ruptura de las membranas se produce de forma espontánea durante el parto, a los 4 centímetros de dilación del cérvix y coincidiendo habitualmente con el aumento de presión intrauterina causada por la contracción. Cuando la bolsa amniótica se rompe, en otras circunstancias de la citada anteriormente, hay que valorar la presencia de algún proceso que las debilite, exceptuando una agresión mecánica como puede ser amniocentesis o la amnioscopia.

La causa de la ruptura prematura de membranas es multifactorial y varía con la edad gestacional. En algunas pacientes más de una posible causa es encontrada. A medida que la ruptura ocurre a menor edad gestacional, se observa una mayor asociación con la infección del corion/decidua 7 aunque, en algunos casos, es difícil saber si la infección es la causa o es secundaria a la ruptura de las membranas. Además del factor arriba mencionado, existen otros factores asociados a ruptura prematura de membranas que son: Bajo nivel socioeconómico, fumar, enfermedades adquiridas por transmisión sexual, parto pretérminos anteriores, labor de parto pretérmino en semanas anteriores en el actual embarazo, conización cervical por tratamientos al cuello del útero, polihidramnios, embarazos múltiples, amniocentesis, cerclaje del cuello del útero y sangrados vaginales durante el actual embarazo, enfermedades pulmonares durante el embarazo, bajo índice de masa corporal, (Vigil et al, 2011).

Según Drife (2005) refiere que la infección intrauterina es el principal factor causal, en el presente caso de estudio nos damos cuenta que la paciente tiene un examen de orina alterado, es decir que tiene ITU, significa que la causa del RPM es la ITU.

\section{Diagnóstico}

La RPPM ocurre en cerca del 3\% de todos los nacimientos, sin embargo, en embarazo de término los porcentajes son muchos más altos y las complicaciones mínimas. La ruptura de membranas puede documentarse utilizando varias técnicas diagnósticas, según Mercer (2003).

Clínica, o visualización de la salida de líquido amniótico transcervical o la acumulación de líquido en fondo de saco vaginal aunado a la historia de la paciente de pérdida transvaginal de líquido confirma el diagnóstico de ruptura de membrana en cerca del $90 \%$ de los casos.

Ecografia, cuando no se visualiza la salida de líquido amniótico transcervical y hay historia de salida de líquido. En estos casos la sospecha de ruptura de membranas aumenta ante la presencia de oligoamnios, sin embargo, no se puede con solo observar el líquido amniótico disminuido confirmar la ruptura de membranas.

Prueba con Nitrazina, consiste en usar un papel amarillo preparado para tal fin, donde lo que se cuantifica es el cambio del PH normal de la vagina (4.5-6.0) y al colocarle el líquido, que sospechamos se torna de color azul/púrpura (ph 7.1-7.3), confirmando la presencia de líquido amniótico. Esta prueba puede presentar falsos positivos ante la presencia de sangre, semen o por la presencia de vaginosis bacteriana.

Prueba de arborización en Helecho, se coloca el líquido existente en el fondo del saco vaginal o de las paredes lateral de la vagina y se observa en forma de helechos al microscopio. Puede ser un falso positivo si se incluye moco cervical. 
Fibronectina Fetal, la determinación de fibronectina fetal a nivel cervico-vaginal ha sido recomendada por algunos investigadores, sin embargo, por sus altos falsos positivos no es recomendado como prueba para diagnosticar ruptura de membranas ovulares.

Una vez diagnosticada la RPM, es muy importante la precisión de la edad gestacional, la determinación del bienestar fetal y la ausencia de corioamnionitis (Martinez et al 1998)

\section{Decisión terapéutica}

En la actualidad no existe un tratamiento establecido. Se intenta tratar las consecuencias derivadas de esta circunstancia, en función de los riesgos maternos y fetales. A continuación se detalla el tratamiento según (Vivanco, 2012).

Normas generales para todas las gestantes

Al ingreso se realizará: historia clínica completa, constantes maternas (temperatura, pulso y tensión arterial), exploración obstétrica básica, valoración cervical mediante visualización con espéculo registro cardiotocográfico basal (RCTGB), ecografía (tamaño fetal, edad gestacional, descartar presencia de malformaciones, valoración de LA.), extracción de sangre: hemograma, determinación de proteína $\mathrm{C}$ reactiva (PCR), coagulación, tomas para urocultivo y cultivo vagino-rectal para la identificación del estreptococo del grupo B.

Controles periódicos: toma de temperatura $\mathrm{y}$ pulso, RCTGB se repetirá 2 veces al día, durante los tres primeros días, hemograma y PCR, 1 vez al día.

Si los controles anteriores han salido normales, el RCTGB se realizará 1 vez al día, los análisis cada 2 días y la ecografía cada semana para ver la evolución del LA. Si existieran dudas sobre la existencia de una infección, se realizará un perfil biofísico fetal para comprobar su estado (Gonzales, 2006).

Si existen signos de corioamnionitis o hipoxia fetal, en función de las características de cada centro, se valorará la vía más adecuada de finalización de la gestación, en relación a la viabilidad fetal e interés materno-fetal.

Desde el punto de vista de la madurez pulmonar, la seguridad en el diagnóstico y la gravedad de la afectación fetal, se podrá valorar completando el tratamiento con corticoides para finalizar la gestación. (Cararach, 2006).

\section{Tratamiento medicamentoso}

Según la Federación Latinoamericana de Sociedad Española de Ginecología (2011).

\section{Antibióticos en la RPM.}

- Ampicilina 2gr IV/6h + Eritromicina 250 mgIV/6h durante 48 horas, seguido de 5 días con Amoxicilina y Eritromicina oral.

- Eritromicina $250 \mathrm{mg}$ oral/6h durante 10 días.

- Eritromicina $250 \mathrm{mg}$ IV/6h durante 24 horas, seguido de Eritromicina $500 \mathrm{mg}$ oral/6h hasta una semana.

- Ampicilina 2 gramos iniciales seguido de 1 gr/4h durante 3 días y seguir con:

1 gr/8h de Amoxicilina + Clavulánico, durante 5-10 días.

- Ampicilina 1 gr IV/6h durante 24 h, seguido de Amoxicilina $500 \mathrm{mg} / 8 \mathrm{~h}$ oral hasta una semana o hasta recibir el resultado negativo del cultivo de Estreptococo del grupo B.

\section{Tratamiento con corticoides}

- La terapia con corticoides ha demostrado acelerar la maduración pulmonar fetal.

- Se recomienda la administración a la mujer de Betametasona 12 mg/día IM, durante 2 días.

- Tratamiento con tocolíticos la valoración se realizará de forma individualizada según la situación clínica de la mujer. (Gilbert, 2003).

- Actitud terapéutica según semanas completas de gestación.

- Se detallará la actitud terapéutica según Dare citado por Vivanco y Mori. (Vivanco, Mori, 2012).

\section{Gestaciones de 35 o más semanas de gestación}

A partir de las 34 semanas la finalización de la gestación, ha demostrado tener mejores resultados que el tratamiento conservador. Los corticoides no tienen indicación.

En función del índice de Bishop se puede esperar un período de 12 a 24 horas para que el parto se inicie espontáneamente, o inducirlo mediante la utilización de oxitocina IV o prostaglandinas, según la situación.

\section{Gestaciones de 26 a 34 semanas}

Según el protocolo de la S.E.G.O. se debe de valorar de forma individualizada el riesgo de prematuridad y de infección. El tratamiento conservador con el objetivo de continuar, en la medida de lo posible, la gestación estaría indicado hasta que se compruebe la madurez fetal, aparezcan complicaciones que 
aconsejen finalizar el embarazo, el riesgo de infección supere el riesgo de prematuridad, se alcance la semana 34 (aunque podría adelantarse en función de los resultados perinatales de cada hospital).

El reposo en cama, la administración de corticoides durante 48 horas y los antibióticos profilácticos mejoran los resultados maternos y fetales. Un vez que se decide finalizar la gestación, la extracción fetal se realizará por la vía más segura. (Burroughs, 2002).

\section{Por debajo de las 26 semanas de gestación}

El tratamiento será expectante hasta la semana 31, similar al descrito anteriormente. El riesgo de secuelas importantes es elevado y las probabilidades de supervivencia disminuyen. Se valorará de forma específica cada circunstancia.

\section{Proceso de Atención de Enfermería}

\section{Valoración}

Según patrones funcionales de Marjori Gordon (NANDA, 2012)

Datos significativos: Por patrones funcionales.

\section{Patrón percepción control de la salud}

- Antecedentes de fertilización in vitrio.

- Cesárea anterior (2008) RN nació prematuro y murió a los 7 días.

- Antecedentes de preeclampia en embarazo anterior.

- Posoperada de colecistectomía año 2007.

- Poscesareada 2008.

- Tuvo Hepatitis A hace 4 años.

- Gastritis hace 5 años.

- Tomó Ciprofloxaciono 1 pastilla cada 12 horas por presentar ITU.

- Alérgica al tramal.

- Regular estado de higiene.

- "Deseo saber si el líquido que está eliminando es malo y si le va a afectar a mis bebés".

Patrón Autopercepción autoconcepto-tolerancia situación al estrés

- Ansiosa

- Temerosa

- Presenta nerviosismo.

- Preocupada por pérdida de líquido y estado de salud de sus bebés, situación laboral y alejamiento con su familia por transferencia.
Patrón descanso sueño

- Con problemas para dormir por interrupciones por el personal para el tratamiento y por preocupaciones personales.

\section{Patrón perceptivo cognitivo}

- Paciente quejumbrosa con facies de dolor.

- Refiere presentar dolor a nivel de la cintura y cabeza en un a escala de 4 de la EVA.

\section{Patrón actividad ejercicio}

- FR: $24 \mathrm{x}^{\prime}$

- Respiración profunda

- FC: $110 x^{\prime}$

- PA: $140 / 80 \mathrm{~mm} \mathrm{Hg}$

- Edemas en MM II (+)

- Presenta catéter periférico en MSI

- Capacidad de autocuidado II $^{\circ}$

- Posición trendelenburg

- Reposo absoluto, no puede realizar baño, uso de inodoro

Patrón nutricional metabólico

- Palidez en piel y mucosas.

- Hb: $11.3 \mathrm{gr} / \mathrm{dl}$.

- Peso de $4 \mathrm{Kg}$ en los últimos 12 días

- Apetito aumentado.

- Dentadura incompleta.

- Aumento de $5.5 \mathrm{~kg}$ en 12 días.

- Patrón eliminación.

- Realiza 1 deposición diaria con dificultad.

- Actualmente recolectando orina de 24 horas para proteinuria.

Patrón sexualidad reproducción

- Gestante de 31.6 semanas (embarazo gemelar).

- Mamas sensibles.

- 1 RN prematuro por cesárea que falleció a los 7 días.

- Pérdida de líquido amniótico en regular cantidad (prolongado).

- Última relación sexual hace 2 días.

Exámenes auxiliares

Ecografía Fetos discordantes

PCR: aumentado

TGP: aumentado

\section{Priorización de los diagnósticos encontrados}

- Riesgo de alteración de la diada materno fetal relacionado con complicaciones del embarazo: 
Rotura Prematura de Membranas y gestación múltiple.

- Perfusión tisular periférica ineficaz relacionado con hipertensión arterial, evidenciado por PA de 140/80 mmHg y Edema (+) MMII.

- Riesgo de infección relacionado a presencia de catéter periférico, RPM y disminución de la hemoglobina.

- Dolor agudo relacionado con agentes lesivos (biológicos) evidenciado por conductas expresivas de dolor, máscara facial y FC aumentado.

- Ansiedad relacionado con cambios en el estado de salud, situación económica y laboral evidenciado por expresión de preocupaciones sobre el estado de salud de sus bebés, situación económica y laboral.

- Deterioro de la movilidad física relacionado con prescripción de restricción de movimientos, evidenciado por reposo absoluto secundario a RPM.

- Trastorno del patrón de sueño relacionado con interrupciones por controles y tratamiento evidenciado por insatisfacción del sueño y referencia de pocas horas de sueño.

- Déficit de autocuidado: baño, uso de inodoro relacionado a tratamiento (reposo absoluto) por RPM evidenciado por incapacidad de baño general e incapacidad de uso de inodoro.

- Riesgo de deterioro de la integridad cutánea Relacionado con inmovilización física.

- Riesgo de motilidad gastrointestinal disfuncional relacionado a reposo absoluto y ansiedad.

- Disposición para mejorar la gestión de la propia salud (secundario a controles prenatales regulares y referencia de querer conocer las complicaciones de RPM).

\section{Plan de cuidados de enfermería}

1. Diagnóstico de enfermería: Riesgo de alteración de la diada materno/fetal relacionado con complicaciones del embarazo: Rotura Prematura de Membranas y gestación múltiple.

Definición: Riesgo de alteración de la diada simbiótica materno-fetal como resultado de comorbilidad o condiciones relacionadas con el embarazo.

Dominio 8: Sexualidad

Clase 3: Reproducción

Código: 00209

Página: 331
Objetivo general: Paciente disminuirá riesgo de alteración de la diada materno/fetal, durante el turno.

Intervenciones:

- Controlar funciones vitales (PA,FC, T)c/4 horas.

- Control de LCF C/4 horas.

- Valorar perdida de líquido amniótico verificando, cantidad.

- $\mathrm{C} / 4$ horas.

- Mantener a la paciente en reposo absoluto, posición trendelenburg.

- Administrar Dexametasona 6mg c/12 IM

- Evitar tacto vaginal constante.

- Educar a la madre sobre los cuidados en paciente con RPM y posibles complicaciones.

- Efectivizar, si fuera necesario, exámenes de ecografía o doppler obstétrico. (Reder, 1995)

Evaluación: Objetivo Parcialmente Alcanzado: Paciente presenta funciones dentro de los parámetros normales (PA, FC, T), los LCF de ambos bebés están dentro de los parámetros normales pero aún presenta pérdida de líquido amniótico.

2. Diagnóstico de enfermería: Perfusión tisular periférica ineficaz relacionado con hipertensión arterial, evidenciado por PA de 140/80 $\mathrm{mmHg}$, Edema (+) MMII.

Definición: Disminución de la circulación sanguínea periférica que puede comprometer la salud.

Dominio 4: Actividad/reposo

Clase 4: Respuesta cardiovascular/pulmonar.

Código: 00204

Página: 239

Objetivo general: Paciente presentará perfusión tisular periférica eficaz, durante el turno.

Resultados esperados: Paciente presentará PA/ dentro de los parámetros normales (100/60-130/80)

Paciente presentará disminución de edemas en MMII

Intervenciones:

- Control de PA c/4 horas.

- Valorar signos premonitores de preeclampsia como zumbido de oído, cefalea, etc.

- Administrar Nifedipino $10 \mathrm{mg}$ VO si $\mathrm{Pa}>$ 140/80mmhg PRN.

- Coordinar con la nutricionista para la administración de dieta hiposódica e hiperproteica.

- Efectivizar Proteinuria de 24 horas.

- Realizar examen de Albuminuria. Diario. 
- Control de peso diario

- Controlar estrictamente el BH cada turno.

- Mantener MMII elevados durante el turno.

- Educar a la paciente sobre los signos premonitores de la preeclampsia y su tratamiento.

Evaluación: Objetivo alcanzado, paciente presenta perfusión tisular eficaz durante el turno, presenta una PA de 110/70 y disminución de edemas en miembros inferiores.

3. Diagnóstico de enfermería: riesgo de infección relacionado con presencia de catéter de vía periférica, RPM y disminución de la hemoglobina.

Definición: aumento del riesgo de ser invadido por organismos patógenos.

Dominio 11: Seguridad/Protección

Clase 1: Infección

Código: 00004

Página: 417

Objetivo general: paciente disminuirá riesgo de infección durante estancia el turno.

\section{Intervenciones:}

- CFV (T y pulso) cada turno.

- Valorar vía periférica en busca de signos de flogosis (color, calor, dolor y tumefacción), durante el turno.

- Valorar signos de anemia (palidez de piel y mucosas, disminución de la Sat Oxigéno, etc).

- Valorar signos de corioamnionitis: dolor en zona pélvica, LCF, FC, c/4hr.

- Observar características de secreción vaginal, olor y color de líquido amniótico cada 4 horas.

- Administrar Ampicilina 1 gr EVC/ 6 hrs, Eritromicina 500mg VO C/ $6 \mathrm{hr}$.
- Realizar higiene perineal con antisépticos en cada turno.

- Canalizar vía periférica con una adecuada técnica aséptica cada 72 horas.

- Mantener vía periférica limpia y con adecuada fijación, en cada turno.

- Administrar medicamentos endovenosos diluidos (por volutrol) cada vez que se administre.

- Administrar suero fisiológico o $\mathrm{Cl} \mathrm{Na} 15 \mathrm{cc}$ después de administrar medicamentos.

- Coordinar con el servicio de nutrición para adecuada ingesta de alimentos ricos en hierro, vitamina $\mathrm{C}$ y proteínas, durante su estancia hospitalaria.

- Administrar sulfato ferroso $300 \mathrm{mg} \mathrm{C} / 24$ horas previa prescripción médica.

- Efectivizar exámenes de laboratorio: hemograma, cuando el médico lo solicite.

- Educar a la paciente sobre la importancia de consumir alimentos ricos en hierro.

Evaluación: Objetivo Parcialmente Alcanzado. Paciente presenta vía periférica libre de infección, con una temperatura de $36.5^{\circ} \mathrm{C}$, con líquido amniótico con características normales, pero aún mantiene hemoglobina disminuida.

\section{CONCLUSIÓN}

Los cuidados enfermeros actualmente se desarrollan siguiendo el proceso enfermero, procedimiento que parte de la aplicación del método científico permitiendo, a los profesionales de enfermería, prestar cuidados de una forma racional, lógica y sistemática, tratándose de un elemento garante de la calidad de los cuidados. De los 9 diagnósticos planteados en el presente caso, 7 fueron alcanzados y 2 fueron parcialmente alcanzados. 


\section{REFERENCIAS BIBLIOGRÁFICAS}

Burroughs. G. Leifer (2002) Enfermería materno infantil Octava Edición. Mexico Editorial, Mac.Graw Hill.

Carpenito L. J. (2013) Manual de diagnósticos enfermeros. $14^{\circ}$ ed. España: Wolters Kluwer.

Cararach Ramoneda V, Tamayo Rojas O, Botet Mussons F. (2006) Rotura prematura de membranas. En: Cabero Roura L. Tratado de Ginecología, Obstetricia y Medicina de la Reproducción. Madrid. Médica Panamericana. p. 598-606.

Drife Janes, Magowan Brian. (2005) Ginecología obstétrica clínica. 1ra ed. España: Elservier.

Dickanson, S. (1995) Enfermería materno infantil. 2da ed. España: Mosby/Doyna.

Federación Latinoamericana de Sociedades de Ginecología y Obstetricia (2011) Guía №1. Guía Clínica Ruptura Prematura de Membranas.

Gilbert ES, Harmon JS. (2003) Manual de embarazo y parto de riesgo. $3^{\mathrm{a}}$ ed. Madrid: Elsevier.

González Bosquet E. (2006) Rotura prematura de las membranas amnióticas. En: González- Merlo J, Lailla Vicens JM, Fabre-González E, González Boaquet E,coordinadores. Obstetricia. $5^{\mathrm{a}}$ ed. Barcelona: Masson. p. $563-76$.
Martinez Gonzalez, Luis Raúl; Valladares Hernandez, Marta Y Villate, Jorge Luis. (1998) Análisis de los casos con rotura prematura de membranas y menos de 34 semanas. Rev Cubana Obstet Ginecol [online]. vol.24, n.3, pp. 145-150. ISSN 1561-3062.

Mercer BM. (2003) Preterm Premature Rupture of the Membranes. Obstet Gynecol.;101:178-93.

NANDA Internacional 2012 - 2014 (2012) Diagnósticos enfermeros: definiciones y clasificación. España: Editorial Elsevier.

Reeder S. (1995). Enfermería Materno Infantil. 17a ed. España: McGraw Hill Interamericana.

Usandizaga M. (2005) Tratado de obstetricia y ginecología. $2^{\circ}$ ed. Madrid. MacGraw-hill-interamericana; p.31728.

Vigil De Gracia Paulino, Savransky Ricardo, Wuff Juan Andrés, Delgado, Gutierrez Julian, Nuñez de Morais Edsón (2011), Guía Clínica de FLAGOS, Rotura Prematura de Membranas. Recurado de: www.sogiba. org.ar/novedades/GC1RPM.pdf.

Vivanco Montes L, Mori Vara Pilar (2012) Plan de Cuidados de la Gestante con Rotura prematura de Membranas Pretérmino. Reduca, serie de trabajos adaptación al grado 4(2):507-546. ISS:1989-5305. 


\section{NORMAS GENERALES PARA PRESENTACIÓN DE ARTÍCULOS}

La Revista Científica de Ciencias de la Salud publica artículos originales empíricos y teóricos, comunicaciones originales cortas, reportes de casos en las áreas de Enfermería, Psicología, Nutrición y Medicina. Todo investigador que desee colaborar en la revista deberá considerar las siguientes indicaciones:

1. Los manuscritos deben enviarse a la dirección de la revista: Universidad Peruana Unión, Altura Km. 19.5 Carretera Central, Ñaña, Lima, Perú, Tel.: 01-6186300, Anexos 3072 - 2227, o escribir al correo electrónico: revistasalud@upeu.edu.pe. Deberán ser originales e inéditos y se asume que toda persona que figure como autor ha dado su conformidad para que el manuscrito sea publicado.

2. Los manuscritos recibidos para publicación son sometidos a un proceso de revisión por pares. El manuscrito es enviado a dos revisores nacionales o extranjeros, pares de los investigadores, quienes realizan las correcciones u observaciones que sean pertinentes; luego el manuscrito es devuelto a los autores para que realicen las correcciones o respondan a las observaciones realizadas. En el caso que los revisores emitan opiniones contradictorias, se envía a un tercer revisor. Luego, el manuscrito es sometido a una revisión de la redacción y finalmente pasa a la diagramación. La versión diagramada tal como será publicada se envía a los autores para su corrección o aprobación.

3. El manuscrito debe ser presentado mediante una carta dirigida al editor jefe de la Revista Científica de Ciencias de la Salud, firmada por uno de los autores, solicitando la evaluación para ser considerada su publicación. La carta debe incluir el título del trabajo, el nombre completo de los autores y tipo de trabajo. Junto con la carta de presentación se debe entregar un original impreso, y la versión electrónica del artículo en CD-R.

4. Se debe adjuntar una declaración jurada firmada por todos los autores (DECLARACIÓN JURADA Autores de artículos), que el artículo presentado es propiedad intelectual de los autores, que no ha sido publicado, ni presentado para evaluación en otra revista, cediendo los derechos de autor a la Revista Científica de Ciencias de la Salud una vez que el artículo sea publicado.

5. El artículo debe ser redactado en castellano, portugués o inglés, impreso en papel bond blanco de medida ISOA4 $(212 \times 297 \mathrm{~mm})$, en una sola cara, tipo de letra Times New Roman, tamaño de fuente 12 picas, a doble espacio, con márgenes de $25 \mathrm{~mm}$. Cada parte del artículo debe empezar en página aparte, numeradas en forma consecutiva. En el caso de citas textuales, el párrafo correspondiente dentro del texto se destacará colocándolo entre comillas o escribiéndolo con letra itálica. Las tablas, gráficos y figuras con su título correspondiente, se colocan al final del texto en páginas aparte; no deben ser insertados en el texto.

\section{ESQUEMAS DE PRESENTACIÓN:}

Investigaciones originales: Resumen; Abstract; Introducción; Material y métodos; Resultados; Discusión; Referencias bibliográficas; Tablas y figuras. La extensión total del manuscrito, incluyendo las referencias bibliográficas, no debe ser mayor de 14 páginas escritas en una sola cara sin incluir tablas, gráficos y figuras. Se acepta como máximo de diez tablas y figuras; el número máximo de referencias bibliográficas es 40 .

Comunicaciones cortas: Contienen las mismas partes que una investigación original. La extensión total del manuscrito, incluyendo las referencias bibliográficas, no debe ser mayor de 6 páginas escritas en una sola cara, sin incluir tablas y figuras. Se acepta como máximo de seis tablas y figuras; el número máximo de referencias bibliográficas es 20 .

Reporte de casos: Resumen, Abstract; Introducción, Presentación del caso, Conclusiones, Referencias bibliográficas. La extensión total del trabajo, incluyendo las referencias bibliográficas, no debe ser mayor de seis páginas escritas en una sola cara, sin incluir tablas, gráficos y figuras. Se acepta como máximo de seis tablas, gráficos y figuras; el número máximo de referencias bibliográficas es 20 .

7. Los resúmenes (en el idioma original e inglés) se presentan cada una en hoja aparte, teniendo una extensión máxima de 200 palabras y deben ser escritos en un solo párrafo. Al final se deben agregar 3 a 5 palabras clave o key words, que ayuden a clasificar el artículo. 
8. La información a los autores e instrucciones para la presentación de manuscritos, donde se detallan las normas de formato y contenido de los artículos que se presentarán a la Revista, se pueden obtener de la página electrónica: http://revistasinvestigacion.upeu.edu.pe/index.php?journal=rc_salud

9. El estilo de la revista se basa en la $6^{\circ}$ edición revisada del Manual de Publicaciones de la APA.

10. Una vez que el autor ha sido notificado acerca de la aprobación de su trabajo para su publicación, no se administrarán modificaciones ni agregados.

11. Los nombres y direcciones de correo introducidos en esta revista se usarán exclusivamente para los fines declarados por esta revista y no estarán disponibles para ningún otro propósito u otra persona.

12. Política de acceso abierto: Esta revista provee acceso libre inmediato a su contenido bajo el principio de que hacer disponible gratuitamente la investigación al público, siempre y cuando cite correctamente dentro de su manuscrito, el articulo consultado, lo cual fomenta un mayor intercambio de conocimiento global. 